combination therapies (ACT) are a cornerstone of our strategy for controlling and eventually eliminating malaria. However, reduced responsiveness/resistance to artemisinin derivatives and to ACTs, an increasing problem in South-East Asia is a major concern. It is of utmost importance to develop new antimalarial drugs from novel chemical classes that can replace ACTs. KAF156, an imidazolepiperazine, is a leading candidate in the antimalarial drug development pipeline. Combination of KAF156 with a Solid Dispersion Formulation of lumefantrine (LUM-SDF) is expected to be fast acting, fully curative, improve patient adherence and can potentially reduce malaria transmission.

Methods WANECAM II proposes to advance the clinical development of KAF156 through clinical trials in adults and children, with integrated capacity building and infrastructure development activities. The trial programme will be undertaken in the context of networking, team-building, leadership development and community engagement schemes that will involve intra-European, European-African and intra-African collaborative activities. WANECAM II will accelerate the clinical study of children less than 2 years of age which are the key target for new antimalarial treatments.

Results By the end of the project, the results are expected to contribute to the registration of KAF156/LUM-SDF through stringent regulatory health authorities, increase biomedical research capacity in the consortium and effectively promote networking among the respective teams. A new clinical research team in Niger, a grossly underrepresented country in the African research landscape, will be developed and further increase capacity and infrastructure in the consortium.

Conclusion Providing a new antimalarial drug combination that does not contain an artemisinin derivative and is effective against resistant $P$. falciparum strains as well as gametocytes and that is likely to be taken in 3 or fewer single doses will be a major advance in the field. The new combination of KAF156 with LUM-SDF is expected to provide such major advance upon successful conclusion of the WANECAM II project.

\section{OC-8722 THE GENERATION AND TESTING OF A GENETICALLY ATTENUATED MALARIA PARASITE (GAP) VACCINE}

Shahid Khan, on behalf of the PfSPZ-GA1 Vaccine Consortium. Leiden University Medical Center, Netherlands

\subsection{6/bmjgh-2019-EDC.44}

Background Immunisation with radiation-attenuated Plasmodium falciparum sporozoites (SPZ) (Sanaria PfSPZ Vaccine) can protect $>90 \%$ of vaccinees against controlled human malaria infection (CHMI) and protects against naturally-transmitted P. falciparum in Africa for at least 6 months. Immunisation with sporozoites of genetically attenuated parasites (GAPs), which completely arrest after liver-cell invasion can be potentially safer and more potent than irradiated sporozoite vaccines. As part of a collaboration between two Dutch research groups (LUMC and Radboud University) and the US company, Sanaria, we describe the generation and first-in-man testing of a GAP vaccine,

Methods We screened single and multiple gene deletion parasites in order to identify parasites that can invade hepatocytes but are unable to complete liver-stage development. Informed by rodent studies we created $P$. falciparum double gene- deletion mutant, $\Delta b 9 \Delta$ slarp; sporozoites of this line were infective to human hepatocytes in vitro and to humanised mice but they completely arrest after invasion. Pf $\Delta$ slarp $\Delta b 9$ PfSPZ (Sanaria PfSPZ-GA1 Vaccine) was manufactured in compliance with cGMPs and released for human clinical trials in the EU under a conditional release GMO license. This GAP vaccine was used to perform phase I (safety) and phase $2 \mathrm{a}$ (efficacy) clinical CHMI trial in the Netherlands.

Results In a dose escalating phase I clinical trial, the vaccine showed an excellent safety profile. All adverse events related to the vaccine were mild (grade 1). Based on this indication of safety, vaccine efficacy was examined by CHMI; 48 subjects were subsequently enrolled into phase $2 \mathrm{a}$ study and were immunised with either PfSPZ-GA1 or PfSPZ Vaccine or saline placebo.

Conclusions In conclusion, PfSPZ-GA1 Vaccine is the first injectable genetically attenuated malaria vaccine assessed in humans. The accomplishment to manufacture, obtain regulatory approval and to demonstrate an excellent safety profile for this vaccine is unprecedented and holds a promise for PfSPZ vaccines with increased potency.

\section{OC-8723 CREATING AND ENHANCING TRUSTWORTHY, RESPONSIBLE AND EQUITABLE PARTNERSHIPS IN INTERNATIONAL RESEARCH}

Michelle Singh*, Michael Makanga, on behalf of the TRUST global ethics consortium EDCTP (South Africa). EDCTP Secretariat, The Hague, Netherlands and Cape Town, South Africa

10.1136/bmjgh-2019-EDC.45

Achieving equity in international research remains a crucial concern of the 21st century. Despite initiatives by international organisations on governance frameworks and standards to guide research conduct, such efforts remain disparate and lack focus. In an interdisciplinary collaboration between multi-level ethics bodies, policy-advisors, civil society, funders, industry and academic scholars, the TRUST project combines longstanding, highly respected efforts to establish international governance structures along with networking opportunities between Europe, sub-Saharan Africa and Asia.

The goal of TRUST is to catalyse a collaborative effort to improve adherence to high ethical standards in global research. The strategic outputs of the project encompass a set of ethics tools developed through participatory engagement traversing all continents: (i) a global code of conduct for funders, (ii) a fair research contracting web-tool (iii) and a compliance and ethics follow-up tool.

Since project inception in October 2015, the TRUST consortium has a) created an international network on global research ethics governance to identify generic risks of exporting non-ethical practices; b) established funder and industry platforms; c) identified typical case studies of exporting nonethical practices and reported on lessons learnt; d) developed a global code of conduct that will be used by the European Commission and like-minded funders to foster ethical research and equitable partnerships; e) designed an online Fair Research Contracting tool to empower vulnerable populations under non-ideal conditions; and f) drafted a compliance and ethics follow-up tool, for conditions of high vulnerability.

TRUST envisages to make a tremendous impact on three major issues: a) enhancing the rights of indigenous people who have borne a disproportionate burden of research whilst 
being potentially highly vulnerable to exploitation; b) increasing civil society engagement and improving uptake of the ethics dimension in research and innovation; and c) improving global awareness of the highest ethical standards along with clear, defined incentives for research competitiveness.

\section{OC-8724 NEW VACCINES FOR A SAFER WORLD}

Nathalie Imbault*, Rachel Grant. CEPI, Oslo, Norway

10.1136/bmjgh-2019-EDC.46

CEPI is an innovative partnership between public, private, philanthropic and civil organisations founded in Davos in 2017 to develop vaccines to stop future epidemics. To date, CEPI has received multi-year funding from Norway, Germany, Japan, the Bill \& Melinda Gates Foundation, and the Wellcome Trust. CEPI has also received single-year investments from the governments of Australia, Belgium, and Canada. It has reached $\$ 630$ million of its $\$ 1$ billion funding target. The European Commission has announced a contribution in kind of $€ 250$ million that will support relevant projects through EC mechanisms. Since its launch in January 2017, CEPI has announced two Calls for Proposals. The first was for candidate vaccines against MERS-CoV, Nipah and Lassa viruses. The second was for the development of platforms that can be used for rapid vaccine development against unknown pathogens. From the first call, CEPI has, so far, announced six partnership agreements and this means 4 lassa vaccine candidates, 3 MERS-CoV vaccine candidates and 1 Nipah vaccine candidate have been selected for development. Learn more at CEPI.net. Follow us at@CEPIvaccines.

\section{ABSTRACTS OF POSTER PRESENTATIONS}

\section{PO 7139 CONDITIONAL ECONOMIC INCENTIVES AND MOTIVATIONAL INTERVIEWING TO IMPROVE ADOLESCENTS' RETENTION AND ADHERENCE TO ANTIRETROVIRAL THERAPY IN NIGERIA: ARA TRIAL}

${ }^{1}$ Obinna Ekwunife, 'Maureen Anetoh, ${ }^{2}$ Stephen Kalu, 'George Eleje. 'Nnamdi Azikiwe University, Awka, Nigeria; ${ }^{2}$ Nnamdi Azikiwe University Teaching Hospital, Awka, Nigeria

10.1136/bmjgh-2019-EDC.47

Background Adolescent HIV patients have worse treatment outcomes compared to other age groups given their vulnerability to risk-taking behaviour. Limited evidence exists on the effectiveness of service delivery interventions to support adolescents' retention in care and adherence to antiretroviral therapy (ART). The ARA trial tackles this challenge by evaluating the impact of conditional economic incentives coupled with motivational interviewing on adolescents' retention in care and adherence to ART in Anambra State, Nigeria.

Methods The study will be a cluster-randomised, controlled trial conducted in $12 \mathrm{HIV}$ treatment hospitals in Anambra State, Nigeria. Six (6) hospitals each will be randomised to either intervention or control arm. A structured adherence support scheme, termed the Incentive Scheme, will be applied to the intervention hospitals while the control hospitals will receive routine HIV care. Additionally, patients in the intervention arm will receive motivational interviewing at baseline and following initiation of ART, they will receive a gift voucher of Nigerian Naira (NGN) 2000 when viral load (VL) is $<20$ copies $/ \mathrm{mL}$ at 12 weeks, gift voucher of NGN 1000 if the VL remains suppressed for the next 3 months, and the next 6 months, and finally gift voucher of NGN 2000 if the $\mathrm{VL}$ remains $<20$ copies/mL at 1 year. All gift vouchers will be conditional not only on VL results but also on attending for motivational interviewing. The primary outcome for the trial will be the difference between groups in the proportion to HIV viral load suppression $(\leq 20$ copies/mL) by 12 months and then 24 months after withdrawal of the Incentive Scheme.

Results The protocol for ARA trial and planned activities is finalised. Application for approval for the trial is on-going.

Conclusion The proposed trial will provide evidence on the feasibility of applying the Incentive Scheme to improve retention and adherence to ART of adolescents living with HIV.

\section{PO 7151 COMPARING TWO BED NET DELIVERY MODELS IN RURAL DISTRICTS OF MOZAMBIQUE}

Jorge Arroz. Mozambique Medical Council, Maputo, Mozambique

\subsection{6/bmjgh-2019-EDC.48}

Background The use of long-lasting insecticidal nets (LLINs) is associated with a reduction in malaria transmission. In 2015, a new delivery strategy (intervention) for universal coverage campaign was tested and compared with standard strategy (control). The objective is to compare two bed net delivery models in rural districts of Mozambique.

Methods Two districts served as intervention, and two as control. The following study design was used: 1) before and after; and 2) cost-effectiveness analysis. Three core implementation strategies were tested: 1) use of coupons during household registration, 2) use of stickers to identify registered houses, and 3) a new LLINs allocation criterion. The main endpoints measured were: i) percentage of distributed LLINs; ii) LLINs ownership and use coverage; iii) percentage of households that achieved universal coverage; iv) incremental cost-effectiveness ratio (ICER); v) incremental net benefit (INB).

Results Approximately 88\% $(302,648)$ of LLINs were distributed in intervention districts compared to $77 \%(219,613)$ in control districts [OR: 2.14 (95\% CI: 2.11-2.16)]. Six months after the 2015 campaign, $98.8 \%$ of the 760 households surveyed in the intervention districts had at least one LLIN; $89.6 \%$ of the 787 households surveyed in the control districts had at least one LLIN [OR: 9.7, (95\% CI: 5.25-22.76)]. Near $95 \%$ and $87 \%$ of respondents who had at least one LLIN, reported having slept under the LLIN the previous night in the intervention and control districts, respectively [OR: 3.2; (95\% CI 2.12-4.69)]; 71\% of the households surveyed achieved universal coverage in the intervention districts against $59.6 \%$ in the control districts [OR: 1.6; $(95 \% \mathrm{CI}$ : 1.33-2.03)]. ICER per distributed LLIN was US\$ 0.68. INB was positive.

Conclusion Intervention districts had greater LLINs availability, greater LLINs ownership and use coverage, and a better progression toward reaching universal coverage targets. The new strategy was more cost-effective than the previous strategy. 